\title{
A measurable force driven by an excitonic condensate
}

T. Hakioğlu, Ege Özgün, and Mehmet Günay

Citation: Appl. Phys. Lett. 104, 162105 (2014);

View online: https://doi.org/10.1063/1.4873377

View Table of Contents: http://aip.scitation.org/toc/apl/104/16

Published by the American Institute of Physics

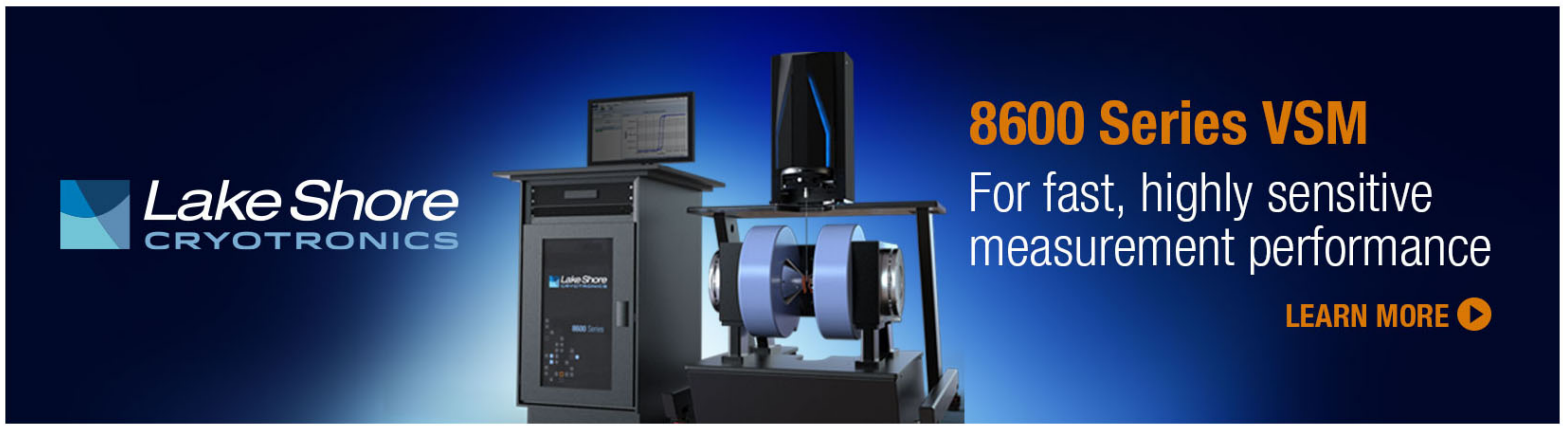




\title{
A measurable force driven by an excitonic condensate
}

\author{
T. Hakioğlu, ${ }^{1,2}$ Ege Özgün, ${ }^{1}$ and Mehmet Günay ${ }^{1}$ \\ ${ }^{1}$ Department of Physics, Bilkent University, 06800 Ankara, Turkey \\ ${ }^{2}$ Institute of Theoretical and Applied Physics, 48740 Turunç, Muğla, Turkey
}

(Received 2 January 2014; accepted 15 April 2014; published online 23 April 2014)

Free energy signatures related to the measurement of an emergent force $\left(\approx 10^{-9} \mathrm{~N}\right)$ due to the exciton condensate (EC) in Double Quantum Wells are predicted and experiments are proposed to measure the effects. The EC-force is attractive and reminiscent of the Casimir force between two perfect metallic plates, but also distinctively different from it by its driving mechanism and dependence on the parameters of the condensate. The proposed experiments are based on a recent experimental work on a driven micromechanical oscillator. Conclusive observations of EC in recent experiments also provide a strong promise for the observation of the EC-force. (C) 2014 AIP Publishing LLC. [http://dx.doi.org/10.1063/1.4873377]

In the late 1940s, Casimir predicted an unusual force between two neutral metallic plates held in vacuum. ${ }^{1}$ The Casimir Force (CF) is attractive between the two ideally infinite metallic plates and the Casimir pressure is given by $P_{c}=\mathcal{F}_{c} / A=-\pi^{2} \hbar c /\left(240 D^{4}\right)$ where $A$ is the area of the plates and $D$ is the separation between them. For typical values $A \simeq 1 \mu \mathrm{m}^{2}$ and $D \simeq 100 \AA, \mathcal{F}_{c} \simeq-1.3 \times 10^{-7} \mathrm{~N}$. The early measurements of $\mathrm{CF}$ were done between a metal plate and a metal sphere. ${ }^{2}$ Twelve years ago perfect agreement with the theory was achieved for the original two plate geometry. 3,4

Vacuum is the lowest energy (ground) state of the electromagnetic radiation with zero field strength and nonzero fluctuations. The electromagnetic field is defined by the excitations of the electromagnetic modes above the vacuum. This perception will be useful here where the vacuum is the ground state of a many body interacting excitonic system in the condensed state. The vacuum of the free electromagnetic radiation is smoothly connected with its excitation spectrum and can be reached perturbatively by changing the number of excited modes and other physical parameters. Same thing is also true for the binary liquid mixtures in the critical regime. A force similar to the universal $\mathrm{CF}$, i.e., the Critical Casimir Force $(\mathrm{CCF})$ has been predicted ${ }^{5}$ and measured ${ }^{6}$ in these systems. On the other hand, in many body interacting systems, there are also nonperturbative ground states that can have a finite energy gap in the excitation spectrum. The existence of the finite gap, away from the critical point where the gap vanishes, can prevent small fluctuations at zero temperature. Close to the critical point however, there are predictions of the $\mathrm{CCF}$ in Bose-Einstein condensates (BEC), ${ }^{7}$ but this has not been experimentally verified yet. On the other hand, the Casimir-Polder-like force between a BEC and a semiconductor plane was measured. ${ }^{8}$ In condensed systems with a finite energy gap away from the critical point at sufficiently small temperatures, one may therefore expect that Casimir like effects are strongly suppressed and may not be observed.

The starting point to generalize Casimir's concept here is the dependence of the free energy on the system's boundaries which may be realized in two different ways. One is the Dirichlet or von-Neumann type boundary conditions affecting the critical fluctuations of the order parameter leading to the Casimir-like phenomena discussed above. The second is the strength of the order parameter, and hence the energy gap itself, depending on system's size through the pairing interaction. Here, we concentrate on the latter taking the example of a low temperature condensate of which the pairing strength depends on the physical size, i.e., the spatially indirect Coulomb coupling between electrons and holes confined to two separate quantum wells (the Double Quantum Well (DQW) geometry) as given by $v_{e h}(\mathbf{r})=-e^{2} /$ $\left(4 \pi \varepsilon \sqrt{\mathbf{r}^{2}+D^{2}}\right)$ where $\mathbf{r}=\left(\mathbf{r}_{e}-\mathbf{r}_{h}\right), \quad \mathbf{r}_{e}$ and $\mathbf{r}_{h}$ are the electron-hole (eh) coordinates, $D$ is the separation between the quantum wells, and $\varepsilon$ is the dielectric constant. There are two effects of $v_{e h}(\mathbf{r})$. If $D$ is on the order of an exciton Bohr radius $a_{B}$ (about $100 \AA$ for GaAs based materials), the first effect is the formation of Wannier Mott excitons. Below a certain critical temperature $T_{c}$, the second effect comes into play. In sufficiently low exciton densities $n_{x}$, when excitons act like independent bosons, they are expected to Bose-Einstein condense ${ }^{9}$ with an energy gap depending on the strength of the Coulomb coupling. As $n_{x}$ increases, the excitons start spatially overlapping, with a higher Fermi energy scale than the pairing interaction, moving into a BCS like condensed ground state. This work is focused on the second effect of the Coulomb interaction.

In an exciton condensate (EC), two different types of pairings ${ }^{10}$ are allowed between an electron in an s-like and a hole in a p-like orbital. The bright pairs have opposite eh spins forming bright singlets and bright triplets, whereas the dark triplet has parallel eh spins. The bright states can couple to the radiation field through the recombination and pair creation due to their odd total angular momenta, whereas the dark states do not. However, in reality, the dark and the bright states are mixed. ${ }^{10-12}$ Two dark states can turn into two bright ones by exchanging their electrons or holes within their proper quantum wells (the Pauli exchange). ${ }^{13}$ Therefore, there is always a weak bright component in the ground state by which the photoluminescence experiments can be made. Until recently, these experiments have been inconclusive in probing the EC due to the weakness of the bright contribution. ${ }^{14}$ Recently, a clear evidence was established ${ }^{15}$ by the observation of the interference fringes due to the condensate's macroscopic wavefunction. 
The condensation free energy (CFE) does not differentiate between the dark and the bright components and hence, offers a promising path in providing additional support to the photoluminescence measurements. ${ }^{15}$ In these systems, the CFE depends on the layer separation $D$ through the condensate's order parameter. For smaller $D$, the attractive coupling is stronger and the CFE is lower, pointing at an attractive force between the electron and hole rich quantum wells. This force, which we may coin as the EC-force, is driven by the Coulomb interaction but is only present due to the condensate. We address here three fundamental questions: (1) Can we understand the analytic dependence of the EC-force on the physical parameters?, (2) Is the EC-force measurable under realistic conditions and current experimental accuracy?, and (3) If so, how can we measure it?

The microscopic Hamiltonian is our starting point given in the eh basis $\left(\hat{e}_{\mathbf{k} \uparrow} \hat{e}_{\mathbf{k} \downarrow} \hat{h}_{-\mathbf{k} \uparrow}^{\dagger} \hat{h}_{-\mathbf{k} \downarrow}^{\dagger}\right)$ at a fixed momentum $\mathbf{k}=\left(k_{x}, k_{y}\right)$ by

$$
\mathcal{H}_{\Delta}=\sum_{\mathbf{k}}\left\{\left(\begin{array}{cc}
\epsilon_{\mathbf{k}} \sigma_{0} & \bar{\Delta}_{\mathbf{k}}^{\dagger} \\
\bar{\Delta}_{\mathbf{k}} & -\epsilon_{\mathbf{k}} \sigma_{0}
\end{array}\right)+\epsilon_{\mathbf{k}}^{(-)} \sigma_{0} \otimes \sigma_{0}\right\},
$$

where $\sigma_{0}$ is the $2 \times 2$ unit matrix, $\bar{\Delta}_{\mathbf{k}}$ is the $2 \times 2$ matrix describing the self-consistent and spin dependent order parameter $\Delta_{\sigma \sigma^{\prime}}(\mathbf{k})$ with $\sigma, \sigma^{\prime}=\{\uparrow, \downarrow\}$ as the spin indices, $\epsilon_{\mathbf{k}}=\left(\xi_{\mathbf{k}}^{(e)}+\xi_{\mathbf{k}}^{(h)}\right) / 2$ and $\epsilon_{\mathbf{k}}^{(-)}=\left(\xi_{\mathbf{k}}^{(e)}-\xi_{\mathbf{k}}^{(h)}\right) / 2$ are the single particle energies in terms of the electron and hole single particle energies $\xi_{\mathbf{k}}^{(e)}=\hbar^{2} \mathbf{k}^{2} /\left(2 m_{e}\right)-\mu_{e}$ and $\xi_{\mathbf{k}}^{(h)}=\hbar^{2} \mathbf{k}^{2} /$ $\left(2 m_{h}\right)-\mu_{h}$ parameterized by the electron and the hole band masses $m_{e}, m_{h}$, and the respective chemical potentials $\mu_{e}, \mu_{h}$. We assume that $m_{e}=m_{h}{ }^{16}$ whereas allow, for now, an imbalance between their concentrations. We have then, $\epsilon_{\mathbf{k}}^{(-)}=-\mu_{-}$where $\mu_{-}=\left(\mu_{e}-\mu_{h}\right) / 2$.

The order parameter in Eq. (1) is given by

$$
\Delta_{\sigma \sigma^{\prime}}(\mathbf{k})=-\frac{1}{2 A} \sum_{\mathbf{k}^{\prime}} V_{e h}\left(\mathbf{k}-\mathbf{k}^{\prime}\right)\left\langle\hat{e}_{\mathbf{k}^{\prime} \sigma}^{\dagger} \hat{h}_{-\mathbf{k}^{\prime} \sigma^{\prime}}^{\dagger}\right\rangle
$$

where $A$ is the sample area and $V_{e h}(\mathbf{q})=-e^{-q D} e^{2} /(2 \varepsilon q)$ is the Fourier transform of $v_{e h}(\mathbf{r})$ with $q=\left|\mathbf{k}-\mathbf{k}^{\prime}\right|$ as the eh exchange momentum. The pairing strength is $\left\langle\hat{e}_{\mathbf{k} \sigma}^{\dagger} \hat{h}_{-\mathbf{k} \sigma^{\prime}}^{\dagger}\right\rangle$ $=\Delta_{\sigma \sigma^{\prime}}(\mathbf{k}) /\left(2 E_{\mathbf{k}}\right)\left[f_{+}(\mathbf{k})-f_{-}(\mathbf{k})\right]$ and $f_{\nu}(\mathbf{k})=1 /\left(1+e^{\beta \lambda_{\nu, \mathbf{k}}}\right)$ is the Fermi-Dirac factor with $\beta=1 / k_{B} T$, with $T$ as the temperature. The energy bands of Eq. (1) are time reversal degenerate, ${ }^{10,12}$ with $\nu=(+,-)$ denoting the doubly degenerate upper and the lower excitonic branches. Here, $\lambda_{\nu, \mathbf{k}}=\epsilon_{\mathbf{k}}^{(-)}+\nu E_{\mathbf{k}}$ are the eigenenergies, $E_{\mathbf{k}}=\sqrt{\epsilon_{\mathbf{k}}^{2}+\Delta_{\mathbf{k}}^{2}}$ and $\Delta_{\mathbf{k}}=\left(\left|\Delta_{\uparrow \uparrow}\right|^{2}+\left|\Delta_{\uparrow \downarrow}\right|^{2}\right)^{1 / 2}$.

Equation (2), together with the self energies, constraints on the particle number conservation and the coupling of the bright states to the radiation field, have been numerically solved in Ref. 12 with an observation that the radiation field strongly suppresses the bright contribution, i.e., $\left|\Delta_{\uparrow \downarrow}\right| \ll$ $\left|\Delta_{\uparrow \uparrow}\right|$ which implies that the condensate is dominated by the dark states, i.e., $\Delta_{\mathbf{k}} \simeq\left|\Delta_{\uparrow \uparrow}(\mathbf{k})\right|=\left|\Delta_{\downarrow \downarrow}(\mathbf{k})\right|$.

The second observation was the presence of a sharp phase boundary determined by $n_{x}, n_{-}$, and $D$ between the condensed phase and the incoherent excitonic liquid determined by $\Delta_{\mathbf{k}}\left(T, n_{x}, n_{-}, D\right)=0$. At $T=0$, the numerical solution of $\Delta_{\mathbf{k}}$ resembles the shape of an inverted parabola (Fig. (3) in Ref. 12) as a function of $D_{c}-D$ near $D \simeq D_{c}$ where $D_{c}$ is the critical layer separation for fixed $n_{x}$ and $n_{-}$. Our first goal here is to understand this behavior analytically and calculate the CFE from which an analytic expression is obtained for the EC-force.

The CFE is given by $\Delta \Omega=\Omega_{\Delta}-\Omega_{0} \leq 0$. Here, $\Omega_{\Delta}$ and $\Omega_{0}$ are the total free energies in the condensed and the uncondensed phases, respectively. In an EC, we observe two types of dependence on $D$. The first is the critical thermal fluctuations of the condensate near $T_{c}$. This term is suppressed if $T \ll T_{c}$. The second dependence on $D$ arises from $\Delta_{\mathbf{k}}$ which is essential for the results in this work. On the other hand, the electron and hole self energies are driven by $D$-independent interactions.

The EC-force is given by

$$
\mathcal{F}_{E C}=-\sum_{\mathbf{k}} \frac{\delta \Delta \Omega}{\delta \Delta_{\mathbf{k}}} \frac{\partial \Delta_{\mathbf{k}}}{\partial D},
$$

where $\Omega_{\Delta}$, up to a $\Delta_{\mathbf{k}}$ independent constant, is

$$
\Omega_{\Delta}=\sum_{\nu, \mathbf{k}}\left\{\nu f_{\nu}(\mathbf{k}) \frac{\Delta_{\mathbf{k}}^{2}}{\left(2 E_{\mathbf{k}}\right)}-\frac{\partial}{\partial \beta} \ln \left(1-f_{\nu}(\mathbf{k})\right)\right\} .
$$

Equation (4) reduces, at $T=0$ and $\epsilon_{k}^{(-)}=0$, to the standard expression $\Omega_{\Delta}=-\sum_{\mathbf{k}}\left\{\Delta_{\mathbf{k}}^{2} /\left(2 E_{\mathbf{k}}\right)+E_{\mathbf{k}}\right\}$.

In the light of the previous discussions and at $T=0$, Eq. (2) reduces to a single gap equation ${ }^{12}$

$$
\Delta_{\mathbf{k}}=-\frac{\pi e^{2}}{\varepsilon} \int \frac{d \mathbf{q}}{(2 \pi)^{2}} \frac{e^{-q D}}{q} G_{\mathbf{k}+\mathbf{q}},
$$

where $G_{\mathbf{k}}=\Delta_{\mathbf{k}} F_{\mathbf{k}} / \sqrt{\left(\epsilon_{\mathbf{k}}-\mu_{x}\right)^{2}+\Delta_{\mathbf{k}}^{2}}$ and

$$
\lim _{k \rightarrow 0} F_{\mathbf{k}}=\left\{\begin{array}{ll}
1 & \text { for } \quad \Delta_{0}^{2}+\mu_{x}^{2}<\mu_{-}^{2} \\
-1 & \text { for } \quad \Delta_{0}^{2}+\mu_{x}^{2}>\mu_{-}^{2}
\end{array} .\right.
$$

The first case in Eq. (6) is allowed when there is a high eh imbalance, indicated by a sufficiently large $\mu_{-}$. In this case, no non-zero solution of Eq. (5) exists, which is consistent with Ref. 12. If $\mu_{-}$is weak or zero, a nonzero solution is allowed by the lower case in Eq. (6). Considering $\mu_{-}=0$ the particle number conservation becomes

$$
n_{x}=\frac{1}{A} \sum_{\mathbf{k}}\left(1-\frac{\epsilon_{\mathbf{k}}}{E_{\mathbf{k}}}\right),
$$

which determines $\mu_{x}$. An exact solution of Eqs. (5) and (7) is not possible due to the presence of the momentum dependent interaction $V_{e h}(\mathbf{q})$. Our motivation here is to resort to a proper approximation which can be done near the phase boundary. We also show below that this solution reproduces the basic results of Ref. 12.

The exponential term $e^{-q D}$ in Eq. (5) hints for a proper approximation implying that the leading contribution comes from $q \ll 1 / D$. Expanding $G_{\boldsymbol{k}+\mathbf{q}}$ up to second order in $q$ near $q=0$, we have $G_{\mathbf{k}+\mathbf{q}} \simeq G_{\mathbf{k}}+\nabla_{\mathbf{k}} G_{\mathbf{k}} \cdot \mathbf{q}+G_{\mathbf{k}}^{\prime \prime} q^{2} / 2$. Here, $G_{\mathbf{k}}^{\prime \prime}$ is 
the second derivative of $G_{\boldsymbol{k}+\mathbf{q}}$ at $q=0$. In both expansions, the first order terms in momentum are absent due to the angular symmetry. Using these in Eq. (5), we have a self consistency condition for $\Delta_{0}=\left.\Delta_{\mathbf{k}}\right|_{\mathbf{k}=\mathbf{0}}$ and $\Delta_{0}^{\prime \prime}=\left.\Delta_{\mathbf{k}}^{\prime \prime}\right|_{\mathbf{k}=\mathbf{0}}$ given by

$$
\Delta_{0}=\sqrt{E_{0}^{2}-\mu_{x}^{2}}, \quad \Delta_{0}^{\prime \prime}=-\frac{\Delta_{0}}{\mu_{x}} \frac{\hbar^{2}}{m}, \quad E_{0}=\frac{e^{2}}{2 \varepsilon D},
$$

yielding $\Delta_{\mathbf{k}}=-\Delta_{0} \epsilon_{\mathbf{k}} / \mu_{x}$. The quasiparticle eigenenergies can then be simply expressed as $E_{k}=\sqrt{\epsilon_{\mathbf{k}}^{2}+\Delta_{\mathbf{k}}^{2}}$ $=E_{0}\left|\epsilon_{k}\right| / \mu_{x}$. Equation (8) is an indication that the model can reproduce the sharp phase boundary with the critical layer separation $D=D_{c}$ given by $D_{c}=e^{2} /\left(2 \varepsilon \mu_{x}\right)$ where, using the parabolic approximation in Eq. (7)

$$
\mu_{x}=-\frac{E_{0}}{2}+\sqrt{\left(\frac{E_{0}}{2}\right)^{2}+\frac{E_{0} n_{x}}{\Gamma}}
$$

with $\Gamma=m_{x} /\left(2 \pi \hbar^{2}\right)$ being the two-dimensional density of states with $m_{x}$ as the exciton reduced mass. Eqs. (8) and (9) can be used to find $\Delta \Omega$ in Eq. (4). Expansion of Eq. (8) near $D=D_{c}$ yields the sharp phase boundary as

$$
\Delta_{0} \simeq \alpha \sqrt{1-\frac{D}{D_{c}}} \quad \text { with } \quad \alpha=\sqrt{\frac{4}{3}} E_{0},
$$

which is valid for $D \leq D_{c}$. The $\Delta \Omega$ given by Eq. (4) can be found similarly using Eqs. (8) and (9) as

$$
\Delta \Omega=-\frac{\Gamma}{E_{0}}\left[\mu_{x}\left(\mu_{x}^{2}+\frac{3}{2} \Delta_{0}^{2}\right)-\mu_{0}^{3}\right], \quad D \leq D_{c},
$$

where $\mu_{0}=n_{x} / 2 \Gamma$ is the chemical potential $\mu_{x}$ evaluated at the phase boundary $\Delta_{0}=0$. Using Eqs. (9) and (10), Eq. (11) can be represented at the phase boundary as

$$
\Delta \Omega=-3 \Gamma \mu_{0}^{2}\left(1-\frac{D}{D_{c}}\right), \quad D \simeq D_{c},
$$

predicting a linear dependence with respect to $D(\Delta \Omega=0$ for $\left.D_{c}<D\right)$. A comparison between Ref. 12 in the vicinity of the phase boundary and Eqs. (10) and (12) are shown in Fig. 1. The accuracy of the parabolic approximation in capturing the main features of the numerical calculations in Ref. 12 is quite remarkable. Encouraged by this, now we proceed to the main result of our work, i.e., finding the EC-force. Using Eq. (12) in Eq. (3) we find that

$$
\frac{\mathcal{F}_{E C}}{A} \simeq-\frac{3}{4} \frac{n_{x}^{2}}{\Gamma D_{c}}, \quad D \simeq D_{c}
$$

with $\mathcal{F}_{E C}=0$ for $D_{c}<D$. The Eq. (8) yields $D_{c}$ as

$$
\frac{a_{B}}{D_{c}}=\frac{2 \varepsilon \mu_{0} a_{B}}{e^{2}}=\frac{n_{x} a_{B}^{2}}{2} \text {. }
$$

For a sample size of $A \simeq 10^{3} \mu \mathrm{m}^{2}$ and a typical concentration of $n_{x} \simeq 3 \times 10^{11} \mathrm{~cm}^{-2}$, the EC-force is $\mathcal{F}_{E C} \simeq 10^{-9} \mathrm{~N}$ which is quite measurable within experimentally available precision. However, there is always the static Coulomb force

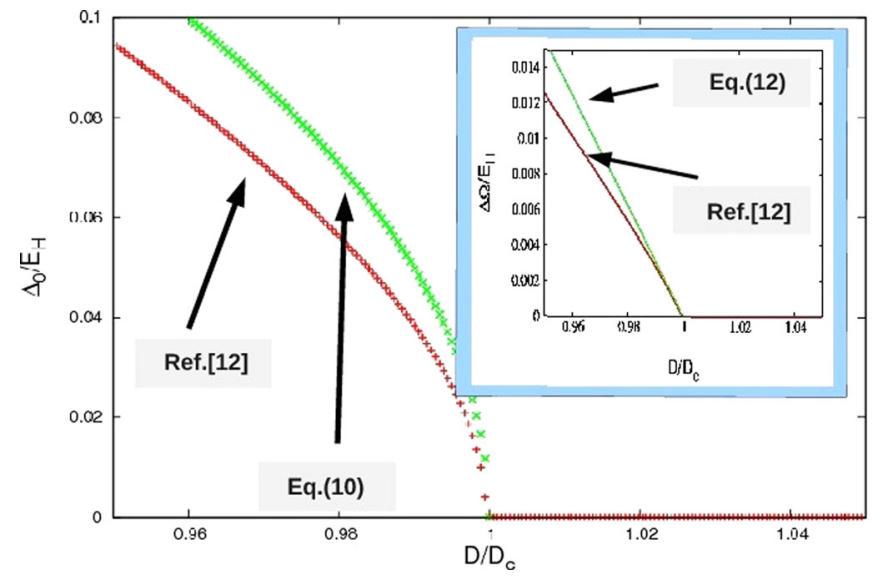

FIG. 1. Comparison of Ref. 12 with Eqs. (10) and (12) as $D$ is varied near $D_{c}$. The main figure depicts $\Delta_{0} / E_{H}$ and the inset is $\Delta \Omega / E_{H}$, where $E_{H}=$ $e^{2} /\left(4 \pi \varepsilon a_{B}\right)$ and $a_{B}=\hbar^{2} 4 \pi \varepsilon /\left(e^{2} m_{x}\right)$. (The numerical solution includes the self energies as well as their realistically different masses.)

present independently from the condensate. One can compare $\mathcal{F}_{E C}$ in the condensed phase with the Coulomb force $\mathcal{F}_{C}$ between the quantum wells. Using Eq. (13) and $\mathcal{F}_{C}=e^{2}$ $n_{x}^{2} A / \varepsilon$ we have

$$
\frac{\mathcal{F}_{E C}}{\mathcal{F}_{C}}=\left\{\begin{array}{lll}
3 a_{B} /\left(8 D_{c}\right) & \text { for } & D_{c}>D \\
0 & \text { for } & D_{c}<D .
\end{array}\right.
$$

Considering $D \simeq 100 \AA$ and $n_{x} \simeq 3 \times 10^{11} \mathrm{~cm}^{-2}$, the two different regimes in Eq. (15) can be controlled by varying $n_{x}$. Note that $\mathcal{F}_{C} \propto n_{x}^{2}$, whereas $\mathcal{F}_{E C} \propto n_{x}^{3}$.

Here, we propose experiments for the measurement of $\mathcal{F}_{E C}$. Due to the dielectric between the quantum wells, the direct measurement is more challenging than measuring the $\mathrm{CF}$ between two metal plates in vacuum. Recently, Yamaguchi, Okamoto, Ishihara, and Hirayama have detected ${ }^{17}$ the motion of a micromechanical oscillator with an amplitude on the order of $50 \mathrm{~nm}$. Upon this work, we use the EC-force as the driver of the micromechanical oscillator as shown in Fig. 2.

In order to measure $\mathcal{F}_{E C}$ one has to subtract the $\mathcal{F}_{C}$ as well as the external electric (E)-field. ${ }^{18}$ For this, we use a

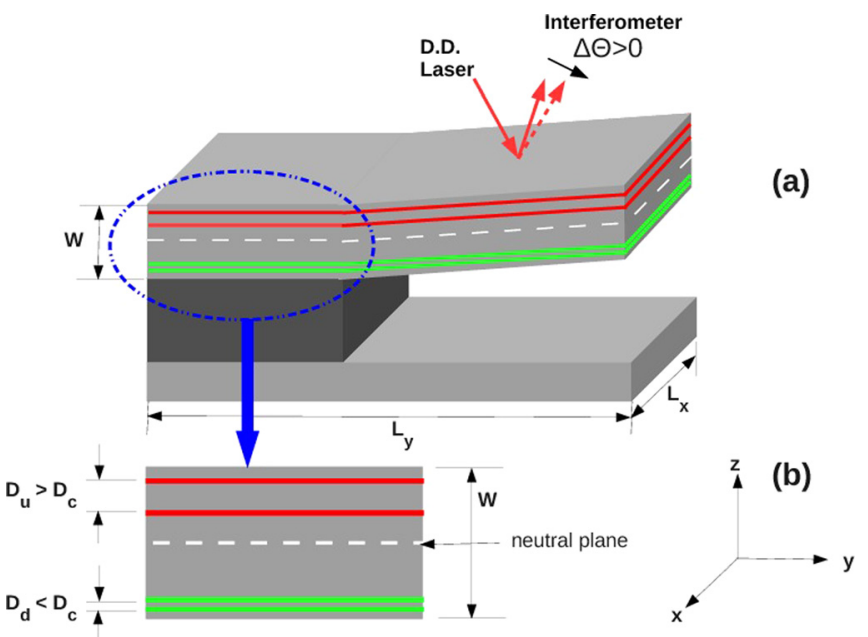

FIG. 2. The proposed mechanical resonator for the EC-force measurement via an interferometer and a DD laser. Here, (a) is the general set-up with physical dimensions, and (b) magnified view of the cross section with two DQWs. 
two DQW geometry and that $\mathcal{F}_{C}=e^{2} n_{x}^{2} A /(2 \varepsilon)$ as well as the external force $\mathcal{F}_{\text {ext }}=e E_{\text {ext }}$ are independent of $D .{ }^{19}$ Specifically, two DQWs with slightly unequal layer separations $D_{u} \neq D_{d}$ are grown on either side of the neutral plane of the cantilever in Fig. 2. The $D_{u}, D_{d}$, and $n_{x}$ are arranged such that one $\mathrm{DQW}$ is driven into the condensed phase, i.e., $D_{d} / D_{c}=1-\delta$, whereas the other is not, i.e., $D_{u} / D_{c}=1$ $+\delta$, where $0<\delta \ll 1$. The DQWs are driven by the same pump laser and are subject to the same external E-field. Using slightly different well widths, the exciton lifetime hence the equilibrium populations in both wells can be made comparable. ${ }^{20}$ In this geometry, the $\mathcal{F}_{C}$ as well as $\mathcal{F}_{\text {ext }}$ in both DQWs are also comparable, whereas, the lower DQW has a nonzero $\mathcal{F}_{E C}$ creating a net bending stress on the cantilever and driving the cantilever's motion as shown below.

Although static measurements can be performed with acceptable accuracy, the measurement of the periodically driven oscillations of the cantilever is more promising. A typical cantilever oscillator ${ }^{17}$ can be driven with a power consumption $P=m_{e f f} \Omega_{0}^{3} \Delta z_{r m s}^{2} / Q \simeq 2 \times 10^{-15} \mathrm{~J} / \mathrm{s}$, where $\Delta z_{r m s}$ is the rms vibrational amplitude, $m_{\text {eff }} \simeq 10^{-10} \mathrm{~kg}$ is the effective mass of the cantilever, $\Omega_{0} /(2 \pi) \simeq 20 \mathrm{kHz}$ is the resonance frequency, and $Q \simeq 2.5 \times 10^{5}$ is the quality factor of the resonator. In Fig. 2(a), geometry at resonance, and for $n_{x}=3 \times 10^{11} \mathrm{~cm}^{-2}$, this leads to $\Delta z_{r m s} \simeq 50 \mathrm{~nm}$. Oscillations within these amplitude and frequency ranges have been measured in Ref. 17. The deflection angle $\Delta \Theta$ can then be measured using a deflection-detection (DD) laser and an optical interferometer. We can estimate $\mathrm{it}^{21}$ as $\Delta \Theta \simeq L_{x} L_{y} \mathcal{F}_{E C} /(12 E I)$, where $E$ is the Young's modulus and $I \simeq L_{y} W^{3} / 3$ is the second area moment. Using $E \simeq 80 \mathrm{GPa}$ for GaAs and $\mathcal{F}_{E C} \simeq 10^{-9} \mathrm{~N}$ for $n_{x}=3 \times 10^{11} \mathrm{~cm}^{-2}$, we find that ${ }^{22} \Delta \Theta \simeq 3 \times 10^{-4} \mathrm{rad}$.

The created electrons and holes reach thermal equilibrium with the lattice within a few ns. A typical driving pulse in mechanical resonance with the cantilever is $10-20 \mathrm{kHz}$ which is much shorter than the lattice thermalization time and much longer than the exciton lifetime avoiding the heating effects. The resulting resonant amplitude, with the quality factor $Q \simeq 2.5 \times 10^{5}$ as in Ref. 17, turns out to be about $50 \mathrm{~nm}$ as given above.

One should also be aware of another secondary effect, i.e., the photon force exerted by the DD-laser in Fig. 2. If a $\mathrm{mW}$ range, $600 \mathrm{~nm}$ wavelength is used for the DD-laser, a simple calculation shows that about $10^{-11} \mathrm{~N}$ photon force would be exerted on the cantilever at normal incidence which can be reduced by another $10^{2}$ times using a wide angle of incidence. The oscillations of the cantilever are however unaffected by this constant force.

In conclusion, the EC created in a DQW, gives rise to a force that is not known yet in other condensed matter systems. Its existence is supported by the recent conclusive observations of EC. ${ }^{15}$ The EC-force, naturally reminds the Casimir effect due to the vacuum fluctuations of the electromagnetic radiation, but its origin is Coulombic although it conceptually differs from the Coulomb force. In the Casimir effect, the driving mechanism is the dependence of the photon density of states on the boundary conditions, whereas in the EC, it is the specific exponential dependence of the Coulomb coupling on the layer separation $D$. As a result, the EC-force depends on the properties of the condensate. In particular, the $1 / D$ dependence in Eq. (13) is in contrast with the $1 / D^{4}$ dependence of the CF.

Finally, we hope that this work can stimulate research in a broader conceptual perspective where a force due to a quantum condensate can be investigated.

The authors are grateful to K.-J. Friedland (Paul-Drude Institute) and A. Dâna (UNAM, Bilkent University), and Nai-Chang Yeh (Caltech) for useful discussions.

${ }^{1}$ H. B. G. Casimir, Proc. K. Ned. Akad. Wet. 51, 793 (1948).

${ }^{2}$ P. H. G. M. van Blokland and J. T. G. Overbeek, J. Chem. Soc., Faraday Trans. 1 74, 2637 (1978); S. K. Lamoreaux, Phys. Rev. Lett. 78, 5 (1997). ${ }^{3}$ G. Bressi, G. Carugno, R. Onofrio, and G. Ruoso, Phys. Rev. Lett. 88, 041804 (2002).

${ }^{4}$ V. M. Mostepanenko and N. N. Trunov, The Casimir Effect and Its Applications (Oxford Science Publications, 1997); K. A. Milton, The Casimir Effect (World Scientific, 2001).

${ }^{5}$ M. E. Fisher and P. G. de Gennes, C. R. Acad. Sci., Paris B 287, 207 (1978). ${ }^{6}$ C. Hertlein, L. Helden, A. Gambassi, S. Dietrich, and C. Bechinger, Nature 451, 172 (2008); M. Fukuto, Y. F. Yano, and P. S. Pershan, Phys. Rev. Lett. 94, 135702 (2005); R. Garcia and M. H. W. Chan, Phys. Rev. Lett. 88, 086101 (2002).

${ }^{7}$ S. Biswas, J. K. Bhattacharjee, D. Majumder, K. Saha, and N. Chakravarty, J. Phys. B 43, 085305 (2010); P. A. Martin and V. A. Zagrebnov, Europhys. Lett. 73, 15 (2006).

${ }^{8}$ J. M. Obrecht, R. J. Wild, M. Antezza, L. P. Pitaevskii, S. Stringari, and E. A. Cornell, Phys. Rev. Lett. 98, 063201 (2007); D. M. Harber, J. M. Obrecht, J. M. McGuirk, and E. A. Cornell, Phys. Rev. A 72, 033610 (2005). ${ }^{9}$ S. A. Moskalenko, Fiz. Tverd. Tela 4, 276 (1962); J. M. Blatt, K. W. Ber, and W. Brandt, Phys. Rev. 126, 1691 (1962).

${ }^{10}$ M. A. Can and T. Hakioğlu, Phys. Rev. Lett. 103, 086404 (2009); T. Hakioğlu and M. Sahin, Phys. Rev. Lett. 98, 166405 (2007).

${ }^{11}$ D. V. Vishnevsky, H. Flayac, A. V. Nalitov, D. D. Solnyshkov, N. A. Gippius, and G. Malpu, Phys. Rev. Lett. 110, 246404 (2013); A. A. High, A. T. Hammack, J. R. Leonard, S. Yang, L. V. Butov, T. Ostatnicky, M. Vladimirova, A. V. Kavokin, T. C. H. Liew, K. L. Campman, and A. C. Gossard, Phys. Rev. Lett. 110, 246403 (2013); A. V. Kavokin, M. Vladimirova, B. Jouault, T. C. H. Liew, J. R. Leonard, and L. V. Butov, Phys. Rev. B 88, 195309 (2013).

${ }^{12}$ T. Hakioğlu and E. Özgün, Solid State Commun. 151, 1045 (2011).

${ }^{13} \mathrm{M}$. Combescot, O. Betbeder-Matibet, and R. Combescot, Phys. Rev. Lett. 99, 176403 (2007).

${ }^{14}$ L. V. Butov, J. Phys.: Condens. Matter 16, R1577 (2004); L. V. Butov, J. Phys.: Condens. Matter 19, 295202 (2007); D. W. Snoke, Adv. Condens. Matter Phys. 2011, 1; D. W. Snoke, Science 298, 1368 (2002).

${ }^{15}$ A. A. High, J. R. Leonard, A. T. Hammack, M. M. Fogler, L. V. Butov, A. V. Kavokin, K. L. Campman, and A. C. Gossard, Nature 483, 584 (2012).

${ }^{16}$ Including realistic values of $m_{e}$ and $m_{h}$ yields a slightly asymmetric shape in the CFE as a function of $n_{-}$. Since $n_{-}=0$ here, the eh mass difference is not crucial for the physics that follows.

${ }^{17}$ H. Yamaguchi, H. Okamoto, S. Ishihara, and Y. Hirayama, Appl. Phys. Lett. 100, 012106 (2012).

${ }^{18} \mathrm{An}$ important issue here is whether the EC-force can move the sample as a whole instead of the eh wavefunctions within the DQWs. The answer is the strong $E_{\text {ext }} \simeq 50 \mathrm{kV} / \mathrm{cm}$ pinning the the eh wavefunctions with an external force $F_{\text {ext }} \simeq 10^{-6} N$. Here, $E_{\text {ext }}$ is also necessary in the experiments for promoting the lifetime of the excitons (see Refs. 14 and 15).

${ }^{19}$ Here corrections to the $\mathcal{F}_{C}$ should be considered. A simple calculation yields $\mathcal{F}_{C}=e^{2} n_{x}^{2} A /(2 \varepsilon)\left(1+2 \sqrt{\pi D^{2} / A}\right)$. For $A$ and $D$ used here, the finite size correction is about $10^{-3}$.

${ }^{20}$ Using the model in Ref. 12, the ratio of the lifetimes in the upper (u) and the lower (d) quantum wells is given by $\tau_{u} / \tau_{d} \simeq \mid\left(D_{d} / D_{u}\right) \exp$ $\left\{-D_{d}^{2} / W_{d}^{2}\right\} /\left.\exp \left\{-D_{u}^{2} / W_{u}^{2}\right\}\right|^{2}$ where $W_{d} \simeq W_{u} \simeq 70 \AA$ as the individual widths of the quantum wells in the upper and the lower DQWs. The widths can be arranged such that $\tau_{u} / \tau_{d} \simeq 1$.

${ }^{21}$ I. S. Sokolnikoff, Mathematical Theory of Elasticity (Mc Graw Hill, New York, 1956).

${ }^{22}$ This is similar to the radian accuracy of the conventional AFMs $\left(10^{-5}\right.$ rad). [D. A. Bonnell, D. N. Basov, M. Bode, U. Diebold, S. V. Kalinin, V. Madhavan, L. Novotny, M. Salmeron, U. D. Schwarz, and P. S. Weiss, Rev. Mod. Phys. 84, 1343 (2012)]. 